\title{
Familial hypokalaemic periodic paralysis in Finland
}

\author{
I M Kantola, L T Tarssanen
}

\begin{abstract}
Seven families with familial hypokalaemic periodic paralysis were found in Finland. Nine of the 103 asymptomatic family members studied had abnormal results on a potassium exercise test. The overall prevalence of familial hypokalaemic periodic paralysis in Finland was $0.4 / 100000$. Carbohydrate intake and hard exercise were the most important triggers of paralytic attacks. Half of the patients reported having attacks at least once a month. Seven patients reported cardiac symptoms (especially bradycardia) during attacks. Permanent muscular weakness was not prominent.
\end{abstract}

Familial hypokalaemic periodic paralysis is a rare disease of undetermined cause characterised by transient attacks of hypotonic paralysis of varying severity, occurring at intervals of days to years in otherwise healthy people. Usually there is hypokalaemia during attacks. Attacks may be precipitated by glucose infusion, high carbohydrate meal, emotional excitement, and severe muscular exertion. The attacks usually begin at puberty and cease at the age of 50-60 years. The mode of inheritance is thought to be autosomal dominant. Attacks are more common in males. ${ }^{1}$

We determined the number of families with hypokalaemic periodic paralysis in Finland and described their characteristics.

\section{Patients and methods}

The number of families with familial hypokalaemic periodic paralysis was determined by using a special questionnaire which was sent to chief physicians in the departments of medicine and neurology in all university hospitals, central hospitals, and district hospitals in Finland. If no response was received hospitals were personally contacted. The family members were interviewed by the investigator. The families were traced as far back as the members could remember. Population registers were not used. A special questionnaire to obtain information about the patient's age at onset, the frequency and severity of paralytic attacks, any possible provocative factors, permanent muscular weakness, and the effect of pregnancy was given to patients with paralytic attacks.

Physical examination was performed on all participants. A potassium exercise test ${ }^{2}$ was carried out on both asymptomatic and symptomatic family members. This is a rectangular 30 minute, $100 \mathrm{~W}$ bicycle exercise test. During the exercise the rise in the plasma potassium concentration is monitored. According to our results, a rise in concentration of $\leqslant 0.5 \mathrm{mmol} /$ 1 or the ratio rise in concentration: pulse rise $<0.090$ suggests the diagnosis of hypokalaemic periodic paralysis.

\section{Results}

Seven families with familial hypokalaemic periodic paralysis were found in Finland. In these families 21 living ( 15 men and six women) and 11 dead (six men and five women) patients with certain paralytic attacks with hypokalaemia were noted. In the seven families 171 living ( 80 men and 91 women) and 119 dead (65 men and 54 women) asymptomatic adult family members were found. Of these 171 family members 103 (49 men and 54 women) were investigated, but not the remainder for various reasons. Nine asymptomatic family members (five men and four women) showed the same low potassium rise in the exercise test as seen in symptomatic family members. ${ }^{2}$ Muscle biopsies were performed on four of them. Typical dilated sarcoplasmic reticulum was seen in all cases.

Most of the patients had been born in central Finland or Savo. Members of two families were born in southwest Finland. The mean (SD) age at onset of paralytic attacks was 12 (5) years (range 6-23 years). This was lower in females $(6.5(0.5)$ years) than in males $(15.5$ (3.5) years). The intensity of the disease seemed to vary from one family to another. One patient had $>9$ attacks a month, three had 4-8 a month, eight had 1-3 a month, two had 1-2 a year, and seven had less frequent attacks.

The attacks began in the legs in five patients, the arms in two, and in both in 14. No facial or eye muscle paralysis was reported. Five patients reported permanent mild muscular weakness in the extremities, which did not affect their ability to work.

One patient had a permanent cardiac pacemaker and aortic valve prosthesis; one other had familial hypercholesterolemia. Physical examination of the other patients revealed no cardiovascular, endocrinological, or gastrointestinal abnormalities. Tendon reflexes were normal. Muscle power was reduced to MRC grade 4 in one patient and normal in all others. 
Five patients experienced constipation during their severest attacks. Three of them had also difficulties in urinating. One patient had polyuria after the attacks. Seven reported cardiac symptoms (especially slow pulse rate) during severe attacks. Five had respiratory symptoms, most of them difficulties in coughing up secretions. One patient described breathing difficulties as if the diaphragm did not move at all.

A variety of factors triggered paralysis. These included carbohydrate intake (11), hard exercise (eight), eating sweets and chocolate (five), evening meals (four), irregular meals (three), cold (two), fatigue (two), and rainy weather (one). One reported an increased frequency of attacks during pregnancy; another had no attacks during pregnancy but had attacks nearly every night after delivery. Three patients had no difficulties during pregnancy and breast feeding.

Twelve patients had used potassium, seven acetazolamide, and two dichlorphenamide as medication. Eight of the 12 patients found potassium useful as a prophylactic agent against attacks, three patients found no difference, and one found it useful only during attacks. Acetazolamide had been useful for five patients as a prophylactic agent. Two patients who had no benefit from acetazolamide were transferred to dichlorphenamide, and their paralytic attacks almost stopped. Four of the 21 patients had not had attacks for 10-20 years. They had had their last attack at the age of 21-40 years. We confirmed the inheritance to be autosomal dominant. The penetrance was weak. The male preponderance was 1.9:1, varying from one family to another.

\section{Discussion}

According to our results the prevalence of familial hypokalaemic periodic paralysis in Finland is $0 \cdot 4 / 100000$. Possibly as knowledge of the disease increases so will the prevalence, as was seen from the Danish numbers. The prevalence in Denmark was calculated as $0 \cdot 8 / 100000$ in 1959 by Sagild $^{3}$ and $1 \cdot 25 / 10000$ in 1978 by Johnsen. ${ }^{1}$ We probably found almost all known Finnish patients because the hospitals where the questionnaire was sent should know all patients with this kind of rare diagnosis. Possibly, however, some patients may be incorrectly diagnosed-for example, some psychiatric diagnosis as seen in Denmark. ${ }^{1}$

Nine asymptomatic family members were found who had abnormal results on the pathological potassium exercise test but no paralytic attacks. Four showed vacuolisation in a muscle biopsy specimen. According to these results about $9 \%$ of asymptomatic family members might be carriers of the hypokalaemic periodic paralysis gene. One of our cases would have been classified as sporadic if the potassium exercise test had not been available. If families are traced back far enough or if accurate enough diagnostic methods for family investigation are used no sporadic cases exist.

We found a preponderance among males, but the ratio seemed to vary considerably from one family to another. In some families the gene was completely non-penetrant in women whereas in another women were affected more than men. This variability may explain the variable penetrance observed in earlier studies. ${ }^{14-6}$ Our families were concentrated mainly in two districts. This concentration may be due to the fact that in the past Finnish people did not move much from one place to another, and the families may have one common progenitor.

The age at onset differed considerably between men and women. Johnsen ${ }^{1}$ did not find this kind of difference in Danish patients. Four of the Finnish patients belonged to the same family, and possibly the early beginning of the paralytic attacks was characteristic to that specific family. The frequency of paralytic attacks may vary not only from one patient to another but also in each patient at different periods of his or her life. ${ }^{17}$ In Finnish patients no variation in paralytic attacks was seen in severe forms of the disease, the patients having had weekly attacks during the whole of their disease. The disease seems to be milder in Finland than in Denmark because about one third of the Finnish patients but only one tenth of the Danish patients ${ }^{1}$ had had only one or two paralytic attacks.

Although many of the patients described slow pulse rate during attacks, only one had had life threatening bradycardia. No other cases of hypokalaemic periodic paralysis with ventricular arrhythmias ${ }^{89}$ were found in Finland.

Kramer et al $^{10}$ suggested that cardiomyopathy might be a complication of hypokalaemic periodic paralysis, but we found no signs of decreased cardiovascular working capacity during the exercise test.

The factors eliciting paralysis were similar in both Finnish and Danish patients. ${ }^{4}$ Patients should be warned against taking large carbohydrate-rich meals before going to bed. Also hard exercise and cold, rainy weather seem to precipitate attacks. No regular changes in the pattern of attacks were seen during pregnancy or breast feeding. There is no reason to advise against pregnancy for women with familial hypokalaemic periodic paralysis.

Almost half of our patients had used potassium prophylactically with good success, although others have reported its failure to prevent attacks. ${ }^{112}$ Its success in our patients may be due to the milder form of the disease in Finland. Acetazolamide also seemed to suppress the paralytic attacks better than potassium, although the patients who used acetazolamide were more seriously affected by the disease. In two patients acetazolamide was not effective and both of them were transferred to dichlorphenamide, which suppressed their attacks almost totally. This confirms an earlier report, ${ }^{13}$ which suggested that if acetazolamide is not effective dichlorphenamide should be used.

The prevalence of permanent muscular weakness varies from one study to another. Baruma et $\mathrm{al}^{4}$ reported some kind of muscular 
weakness in all subjects investigated. It has been shown that if the muscle power decreases, carbonic anhydrase treatment might improve the muscle strength between attacks. ${ }^{114}$ According to subjective information permanent muscular weakness seemed to be no problem in Finnish patients, probably because only half of them had regular annual attacks.

This study was financially supported by grants from City of Turku, Research Foundation of Muscle Diseases, Research Turku, Research Foundation of Muscle Diseases, Research Farmos Oy, and the stipend funds of the University of Turku.

1 Johnsen T. Familial periodic paralysis with hypokalaemia. Dan Med Bull 1981;28:1-27.

2 Tarssanen LT, Kantola IM, Huikko ME. Serum potassium exercise test in the diagnosis of familial periodic paralysis. Acta Neurol Scand 1983;68:30-3.

3 Sagild V. Hereditary transient paralysis with special reference to the metabolism of potassium. Copenhagen: Munksgaard, 1959. Dissertation.
4 Buruma OJS, Bots GTAM, Went LN. Familial hypokalemic periodic paralysis. Arch Neurol 1985;42:28-31.

5 Talbott JH. Periodic paralysis. A clinical syndrome. Medicine (Baltimore) 1941;20:85-143.

6 Woratz G. Hypokalämische paroxysmale Lähmung. Munch Med Wschr 1965;46:2318-23.

7 Zabriskie EG, Frantz AM. Familial periodic paralysis. Bull Neurol Inst NY 1932;2:57-74.

8 Levitt LP, Rose LI, Dawson DM. Hypokalemic periodic paralysis with arrhythmia. $N$ Engl $\mathcal{F}$ Med 1972;286: paralysis

9 Stubbs WA. Bidirectional ventricular tachycardia in familial hypokalaemic periodic paralysis. Proc Roy Soc Med 1976;69:223-4

10 Kramer LD, Cole JP, Messenger JC, Ellestad MH. Cardiac dysfunction in a patient with familial hypokalemic periodic paralysis. Chest 1979;75:189-92.

11 Griggs RC, Engel WK, Resnick JS. Acetazolamide treatment of hypokalemic periodic paralysis. Ann Intern Med 1970;73:39-48

12 Resnick JS, Engel WK, Griggs RC, Stam AC. Acetazolamide prophylaxis in hypokalemic periodic paralysis. $N$ Engl Y Med 1968;278:582-6.

13 Dalakas MC, Engel WK. Treatment of "permanent" muscle weakness in familial hypokalemic periodic paralysis. Muscle Nerve 1983;6:182-6.

14 Thompson AJ, Hutchinson M. Myopathy in hypokalaemic periodic paralysis: reversal with acetazolamide. Ir Med $\mathcal{f}$ periodic paralys 